\title{
A Symbolic Algorithm for Optimal Markov Chain Lumping
}

\author{
Salem Derisavi \\ Department of Systems and Computer Engineering \\ Carleton University, Ottawa, Canada \\ derisavi@sce.carleton.ca
}

\begin{abstract}
Many approaches to tackle the state explosion problem of Markov chains are based on the notion of lumpability, which allows computation of measures using the quotient Markov chain, which, in some cases, has much smaller state space than the original one. We present, for the first time, a symbolic algorithm and its implementation for the lumping of Markov chains that are represented using Multi-Terminal Binary Decision Diagrams. The algorithm is optimal, i.e., generates the smallest possible quotient Markov chain. Our experiments on various configurations of two example models show that the algorithm (1) handles significantly larger state spaces than an explicit algorithm, (2) is in the best case, faster than an efficient explicit algorithm while not prohibitively slower in the worst case, and (3) generates quotient Markov chains that are several orders of magnitude smaller than ones generated by a model-dependent symbolic lumping algorithm.
\end{abstract}

\section{Introduction}

Markov chains (MCs) are among the fundamental mathematical structures used for performance and dependability modeling of communication and computer systems. As the size of an MC usually grows exponentially with the size of the corresponding high-level model, one often encounters the inevitable state explosion problem, which often makes solution of the MC intractable. Many approaches to alleviate or circumvent this problem are implicitly or explicitly based on the notion of lumpability [17, which allows computation of measures of the original MC using the solution of a lumped (or quotient) MC, which, in some cases, is much smaller than the original one.

Even a lumped MC can be extremely large, and therefore, its explicit (e.g., sparse matrix) representation may not fit in memory. Symbolic data structures such as Multi-Terminal Binary Decision Diagrams (MTBDDs) [7] and Matrix Diagrams (MDs) 6] are two of the widely-used approaches that enable us to represent large MCs using less memory than the explicit approach. Nowadays, algorithms that directly generate symbolic representations of MCs from the highlevel model are commonplace.

In one form of classification, there are three types of lumping algorithms: state-level, model-level, and compositional. State-level algorithms work directly

O. Grumberg and M. Huth (Eds.): TACAS 2007, LNCS 4424, pp. 139 154, 2007.

(C) Springer-Verlag Berlin Heidelberg 2007 
Table 1. Examples of previous work on lumping algorithms in probabilistic settings

\begin{tabular}{|c|c|c|c|}
\hline & state-level & model-level & compositional \\
\hline explicit & \begin{tabular}{|l|l|l|} 
& 9 & 5 \\
\end{tabular} & 20 Stochastic Activity Networks & 12 Interactive Markov chains \\
\hline symbolic & 14 & $\begin{array}{l}\text { 10 } \\
18 \\
18 \text { PRISM models }\end{array}$ & $\begin{array}{l}11 \text { Markov chains } \\
\text { represented by matrix diagrams }\end{array}$ \\
\hline
\end{tabular}

on the MC (i.e., at the level of the states) and do not use information from the high-level model. They are optimal, i.e., they generate the smallest possible lumped MC, are restricted neither to a specific high-level formalism nor to a specific type of symmetry, and are often slower than the other two types.

Both model-level and compositional algorithms exploit information from the high-level model specification to generate lumped MCs. Neither types are optimal because the optimal lumping cannot be computed directly from the high-level model. Finally, both types address a specific (set of) formalism(s). Model-level algorithms are distinguished by the fact that they exploit a restricted type of user-specified symmetry while compositional algorithms apply a statelevel algorithm to individual components of a compositional model.

Not all model-level algorithms can automatically find and exploit all types of symmetries. Therefore, the fact that the main source of lumpability is symmetry in the high-level model specification does not imply that model-level algorithms are in general preferred over state-level algorithms, as one may argue. Moreover, there are situations in which only state-level algorithms are applicable. For example, consider an MC that is transformed by a model checking algorithm.

Table 1 shows examples of previous work on lumping algorithms for stochastic/probabilistic models, e.g., Markov chains, Markov decision processes. It classifies them also based on whether they use explicit or symbolic representation. Fairly related to MC lumping, is lumping of non-probabilistic models, a.k.a. bisimulation minimization. Bouali et. al. 2] were the first to apply symbolic BDD-based techniques. Wimmer et. al. 22] improve upon [2] by presenting a general BDD-based algorithm that computes some of the popular bisimulations.

The shaded area in Table 1 indicates where our new algorithm fits. This paper gives, to our knowledge for the first time, a symbolic MTBDD-based MC lumping algorithm and its implementation. In [14, an algorithm based on DNBDDs (Decision-Node BDDs) is given without a concrete implementation or runtime analysis. Our algorithm is (1) symbolic, and hence, it can handle much larger state spaces than explicit algorithms, (2) optimal, i.e., generates the smallest possible lumped MC, (3) state-level, i.e., does not rely on the highlevel model, and (4) faster than the efficient explicit algorithm of [9] in the best case, and not prohibitively slower in the worst case we experimented.

The rest of the paper is organized as follows: Section 2 gives an overview of CTMC\& 1 (Continuous Time Markov Chains), lumpability of CTMCs, the explicit lumping algorithm of [9] which is the basis of our new algorithm, and MTBDDs. Sections 3 and 4 put forward the new contributions of this paper. The former

${ }^{1}$ Although the paper is focused on CTMCs, the algorithms can be adapted for DTMCs (Discrete Time Markov Chains) in a very straightforward manner. 
explains how we transformed the explicit algorithm to a symbolic algorithm that is not so fast. The latter presents two techniques that dramatically improve the running time of our algorithm. In Section 5, we compare the running time of our symbolic algorithm, the explicit state-level algorithm of [9], and the symbolic model-level algorithm of [18] by applying them to several configurations of two example models. We finally conclude in Section 6 .

\section{Background}

\subsection{Notation, CTMC, and Lumpability}

All matrices are real-valued and typeset with bold characters. All sets are finite and typeset with roman characters. We consider a CTMC $M=(S, \mathbf{R})$ with state space $S$ and state transition rate matrix $\mathbf{R}: S \times S \rightarrow \mathbb{R}^{\geq 0}$ where $\mathbf{R}(s, s)=0$ for all $s \in S$. The generator matrix $\mathbf{Q}: S \times S \rightarrow \mathbb{R}$ is defined as $\mathbf{Q}(s, s)=$ $-\sum_{s^{\prime} \in S} \mathbf{R}\left(s, s^{\prime}\right)$ and $\mathbf{Q}(s, t)=\mathbf{R}(s, t)$ for all $s, t \in S$ and $s \neq t$. Let $n=|S|$ and $m$ denote the number of non-zero entries of $\mathbf{R}$. For a matrix $\mathbf{A}$ and $C \subseteq S$, we define $\mathbf{A}(s, C)=\sum_{s^{\prime} \in C} \mathbf{A}\left(s, s^{\prime}\right)$ and $\mathbf{A}\left(C, s^{\prime}\right)=\sum_{s \in C} \mathbf{A}\left(s, s^{\prime}\right)$. Consider a partition $\Pi=\left\{C_{1}, \ldots, C_{\widetilde{n}}\right\}$ of $S$. Sets $C_{1}, \cdots, C_{\widetilde{n}}$ are the equivalence classes, or in short, classes of $\Pi$. We use $[s]_{\Pi}$ to denote the class of $\Pi$ that contains $s \in S$. Partition $\Pi^{\prime}$ is a refinement of $\Pi$ (or finer than $\Pi$ ) if every class of $\Pi^{\prime}$ is a subset of some class of $\Pi$. In that case, $\Pi$ is said to be coarser than $\Pi^{\prime}$.

Often, the final goal of a CTMC analysis is not the computation of the steadystate or transient probability of its states. Instead, it is the computation of highlevel measures such as performability. Many of those high-level measures can be computed using reward values associated with states (i.e., rate rewards) and the stationary and transient probability distribution [16. In this paper, we do not concern ourselves with those details as they do not contribute to the main ideas of our algorithm. However, we will briefly explain how to adapt the algorithm to take rate rewards and initial probability distribution into account.

Sometimes, the desired measures can be obtained from a smaller (lumped) CTMC using less time and space. The lumped CTMC is constructed through a partition (or equivalence relation) on the state space of the original CTMC. For that to be possible, the original CTMC should satisfy a set of conditions with respect to that partition. Following [4, two of the most important sets of conditions (and the types of lumping they lead to) on the generator matrix $\mathbf{Q}$ are outlined in Definition 1. Often, it is necessary to check the lumpability conditions in terms of $\mathbf{R}$ instead of $\mathbf{Q}$. Theorem 1 serves that purpose. Finally, the lumped (or quotient) CTMC is obtained using Theorem 2. For more details on the properties of ordinary and exact lumping see [4].

Definition 1. Consider a $C T M C M=(S, \mathbf{R})$, its corresponding $\mathbf{Q}$ matrix, and a partition $\Pi$ of $S$. Then, with respect to $\Pi, M$ is

1. ordinarily lumpable iff $\forall C, C^{\prime} \in \Pi, s, \hat{s} \in C: \mathbf{Q}\left(s, C^{\prime}\right)=\mathbf{Q}\left(\hat{s}, C^{\prime}\right)$, and

2. exactly lumpable iff $\forall C, C^{\prime} \in \Pi, s, \hat{s} \in C: \mathbf{Q}\left(C^{\prime}, s\right)=\mathbf{Q}\left(C^{\prime}, \hat{s}\right)$. 
Theorem 1 (Theorem 2.1 of [8]). Consider a CTMC $M=(S, \mathbf{R})$. With respect to a partition $\Pi, M$ is

1. ordinarily lumpable iff $\forall C \neq C^{\prime} \in \Pi, s, \hat{s} \in C: \mathbf{R}\left(s, C^{\prime}\right)=\mathbf{R}\left(\hat{s}, C^{\prime}\right)$.

2. exactly lumpable if $\forall C, C^{\prime} \in \Pi, s, \hat{s} \in C: \mathbf{R}(s, S)=\mathbf{R}(\hat{s}, S) \wedge \mathbf{R}\left(C^{\prime}, s\right)=$ $\mathbf{R}\left(C^{\prime}, \hat{s}\right)$.

Theorem 2 (Theorems 2.2 and 2.3 of [8]). Let $C T M C M=(S, \mathbf{R})$ be ordinarily or exactly lumpable with respect to a partition $\Pi$ of $S$. Then $\widetilde{M}=$ $(\widetilde{S}, \widetilde{\mathbf{R}})$ is the lumped (or, quotient) CTMC such that

$$
\begin{aligned}
& \widetilde{S}=\{\text { arbitrary element of } C \mid C \in \Pi\} \\
& \widetilde{\mathbf{R}}\left(\tilde{s}, \tilde{s}^{\prime}\right)=\left\{\begin{array}{lll}
\mathbf{R}\left(\tilde{s},\left[\tilde{s}^{\prime}\right]_{\Pi}\right) & \text { (ordinary) } & \text { if } \tilde{s} \neq \tilde{s}^{\prime} \\
\mathbf{R}\left([\tilde{s}]_{\Pi}, \tilde{s}^{\prime}\right) & \text { (exact) } & \text { if } \tilde{s} \neq \tilde{s}^{\prime} \\
0 & \text { (both) } & \text { if } \tilde{s}=\tilde{s}^{\prime}
\end{array}\right.
\end{aligned}
$$

Note that although $\widetilde{S}$ depends on the arbitrarily selected element of each class of $\Pi$, all possible lumped CTMCs will be "equivalent".

\subsection{Explicit State-Level Lumping Algorithm}

The basis of our new symbolic algorithm is the efficient lumping algorithm of [9]. It is an optimal and explicit state-level algorithm for ordinary lumping. In [8, we extended the algorithm to Markov reward processes (i.e., CTMCs augmented with rate rewards and initial probability distribution) and also to exact lumping. Since we discuss both ordinary and exact lumping, we will use the extended version of 8 in this paper.

Figure 1(a) shows the explicit lumping algorithm. ExpLumPCTMC (ExP stands for explicit) takes the original CTMC $M$ and returns the quotient CTMC $\widetilde{M}$. It works in two stages. First, EXPCOARSESTPART computes the coarsest partition $\Pi$ with respect to which $M$ is lumpable by repetitive refinements of $\Pi{ }^{\text {ini }}$. To extend our algorithm to Markov reward processes we only need to set the initial partition $\Pi^{\text {ini }}$ such that all states with the same value (of rate reward or initial probability) are in the same class. If rate rewards and initial probability distribution are not considered, we set $\Pi^{\mathrm{ini}}=\{S\}$. In the second stage (line 2), EXPCOMPQUOT computes the quotient $\widetilde{M}$ according to Theorem 2 ,

EXPCOARSESTPART maintains $L$, a list of potential splitters. Each refinement iteration of EXPCOARSESTPART (line 3-5) refines $\Pi$ with respect to a potential splitter $B$. ExpSPLIT splits each class $C$ of $\Pi$ into classes $C_{1}^{\prime}, \ldots, C_{\alpha}^{\prime}$ (line 3-4) as follows. For ordinary lumping, the states of $C$ are grouped based on their total outgoing rates to $B$ (line $2_{o}$ of EXPCOMPUTEKEYs) and for exact lumping they are grouped based on their total incoming rates from $B$ (line $2_{e}$ ). More formally,

$$
\forall 1 \leq i, j \leq \alpha, s \in C_{i}^{\prime}, s^{\prime} \in C_{j}^{\prime}: \mathbf{k}(s)=\mathbf{k}\left(s^{\prime}\right) \Leftrightarrow i=j
$$

The algorithm works correctly regardless of the selection of $C_{i}^{\prime}$ in line 5 of ExPSPLIT. If we choose $C_{i}^{\prime}$ to be the largest among $C_{1}^{\prime}, \ldots, C_{\alpha}^{\prime}$, it is proved that the 


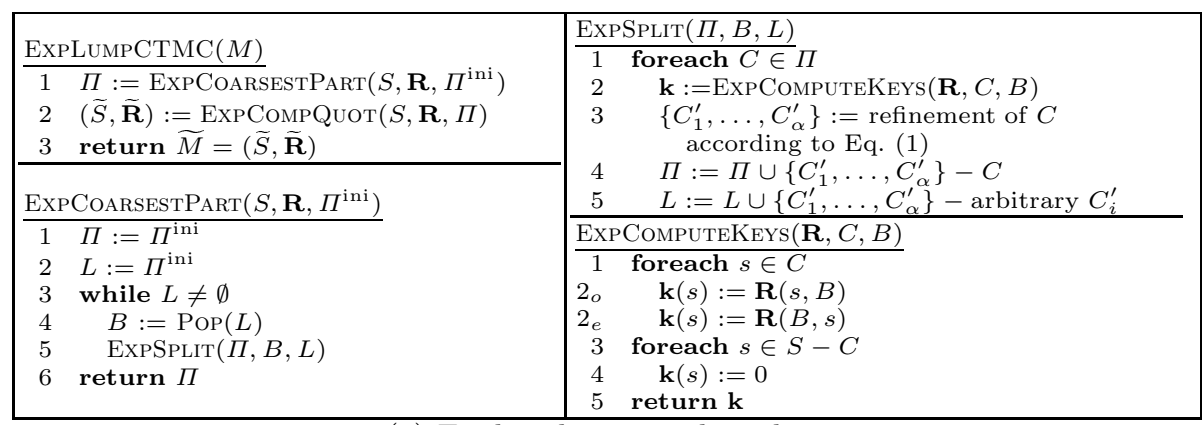

(a) Explicit lumping algorithm

\begin{tabular}{|c|c|}
\hline \multicolumn{2}{|l|}{$\underline{\operatorname{SyMLUMPCTMC}(M)}$} \\
\hline $1 \quad \beta(\Pi):=\operatorname{SymCOARSPART}\left(\mathcal{S}, \mathcal{R}, \beta\left(\Pi^{\mathrm{ini}}\right)\right)$ & $\left.\frac{1}{\mathcal{R}_{0}:=\operatorname{APPLY}(\times, \mathcal{R}, \operatorname{APPLY}}(\times, \mathcal{C}, \operatorname{PERMUTE}(\mathcal{B}))\right)$ \\
\hline $2(\tilde{\mathcal{R}}, \widetilde{\mathcal{S}}):=\operatorname{SyмCомрQuот~}(\mathcal{S}, \mathcal{R}, \beta(\Pi))$ & $1_{e} \mathcal{R}_{e}:=\operatorname{APPly}(\times, \mathcal{R}, \operatorname{APPly}(\times, \mathcal{B}, \operatorname{PeRmUte}(\mathcal{C})))$ \\
\hline $3 \operatorname{return} \widetilde{M}=(\widetilde{\mathcal{R}}, \widetilde{\mathcal{S}})$ & $2_{o} \quad \mathcal{K}_{o}:=\operatorname{SumC}\left(\mathcal{R}_{o}\right)$ \\
\hline SYMCOARSESTPART $\left(\mathbf{R}, S, \beta\left(\Pi^{\text {ini }}\right)\right)$ & $2_{e} \quad \mathcal{K}_{e}:=\operatorname{SumC}\left(\mathcal{R}_{e}\right)$ \\
\hline $\begin{array}{ll}1 & \beta(\Pi):=\beta\left(\Pi^{\mathrm{ini}}\right)\end{array}$ & $3_{o} \quad$ return $\mathcal{K}_{o}$ \\
\hline 2 for $s c:=0$ to $|\Pi|-1$ & $3_{e} \quad$ return $\mathcal{K}_{e}$ \\
\hline $3 \quad \mathcal{B}:=\operatorname{GetClass}(\beta(\Pi), s c)$ & SyMCOMPQuот $(\mathcal{S}, \mathcal{R}, \beta(\Pi))$ \\
\hline $\operatorname{SymSplit}(\beta(\Pi), \mathcal{B})$ & $1 \widetilde{\mathcal{S}}(s):=0 ; \widetilde{\mathcal{R}}(s, t):=0$ \\
\hline return $\beta(\Pi)$ & 2 for $c:=0$ to $|\Pi|-1$ \\
\hline $\operatorname{SyMSPLIT}(\beta(\Pi), \mathcal{B})$ & $3_{o} \quad \mathcal{C}_{c}:=\operatorname{GetCLASS}(\beta(\Pi), c)$ \\
\hline 1 for $c:=0$ to $|\Pi|-1$ & $\mathcal{C}_{c}:=\operatorname{Permute}(\operatorname{GetClass}(\beta(\Pi), c))$ \\
\hline $\mathcal{C}:=\operatorname{GetCLAss}(\beta(\Pi), c)$ & $\mathcal{X}_{c}:=\left\{\right.$ arbitrary element of $\left.\mathcal{C}_{c}\right\}$ \\
\hline $\mathcal{K}:=\operatorname{SymComputeKeys}(\mathcal{R}, \mathcal{C}, \mathcal{B})$ & $\widetilde{\mathcal{S}}:=\operatorname{ApPLY}\left(+, \widetilde{\mathcal{S}}, \mathcal{X}_{c}\right)$ \\
\hline$T:=\{$ leaves of $\mathcal{K}\}$ & $\mathcal{R}^{\prime}:=\operatorname{APPLY}(\times, \widetilde{\mathcal{R}}, \widetilde{\mathcal{S}})$ \\
\hline$\alpha:=1$ & for $c:=0$ to $|\Pi|-1$ \\
\hline foreach $x \in T$ & $\mathcal{R}^{\prime \prime}:=\operatorname{SumC}\left(\operatorname{Apply}\left(\times, \mathcal{R}^{\prime}, \operatorname{Permute}\left(\mathcal{C}_{c}\right)\right)\right)$ \\
\hline $\mathcal{C}_{\alpha}^{\prime}:=\operatorname{ApPLY}(=, \mathcal{K}, x)$ & $\mathcal{R}^{\prime \prime}:=\operatorname{SumR}\left(\operatorname{Apply}\left(\times, \mathcal{R}^{\prime}, \operatorname{Permute}\left(\mathcal{C}_{c}\right)\right)\right)$ \\
\hline$\alpha:=\alpha+1$ & $\mathcal{R}^{\prime \prime}:=\operatorname{Apply}\left(\times, \mathcal{R}^{\prime \prime}, \operatorname{Permute}\left(\mathcal{X}_{c}\right)\right)$ \\
\hline $\operatorname{RePlaCeClass}\left(\beta(\Pi), c, \mathcal{C}_{1}^{\prime}\right)$ & $\widetilde{\mathcal{R}}:=\operatorname{Apply}\left(+, \widetilde{\mathcal{R}}, \mathcal{R}^{\prime \prime}\right)$ \\
\hline $\begin{array}{l}10 \quad \text { for } i:=2 \text { to } \alpha \\
11 \quad \operatorname{ADDCLASS}\left(\beta(\Pi), \mathcal{C}_{i}^{\prime}\right)\end{array}$ & return $(\widetilde{\mathcal{S}}, \widetilde{\mathcal{R}})$ \\
\hline
\end{tabular}

(b) Symbolic lumping algorithm

Fig. 1. Explicit lumping algorithm for Markov chains

algorithm runs in $O(m \lg n)$ time [9]. The algorithm finishes when $\Pi$ is refined with respect to all potential splitters. See [8] for more details.

\subsection{Multi-Terminal Binary Decision Diagram}

BDDs (Binary Decision Diagrams) [3] are a data structure for compact representation of binary functions of $k$ binary variables, i.e., $\{0,1\}^{k} \rightarrow\{0,1\}$. MTBDDs [7] are a variation of BDDs used to represent finite-ranged functions of $k$ binary variables, i.e., $\{0,1\}^{k} \rightarrow A$ where $A$ is a finite set.

MTBDDs are widely used to represent transition matrices of MCs and we follow that in this paper. To that purpose, the MTBDD uses $2 L$ binary variables $v r_{1}, \ldots, v r_{L}$ and $v c_{1}, \ldots, v c_{L}$ that encode the row index and the column index, respectively. Although the variable ordering can be arbitrary, we consider the interleaved ordering in which the top-down order of the variables is $v r_{1}, v c_{1}, v r_{2}, v c_{2}, \ldots, v r_{L}, v c_{L}$. Interleaved ordering often leads to smaller 
MTBDDs for MCs that are generated from high-level models [13. We denote the set of all possible row and column indices (states) by $S_{r}=\chi_{i=1}^{L} v r_{i}$ and $S_{c}=\chi_{i=1}^{L} v c_{i}$. We use calligraphic letters to denote the MTBDD representation of matrices and sets (described below). We denote an element of $\mathcal{R}$ by $\mathcal{R}(s, t)$ where $s \in S_{r}$ and $t \in S_{c}$ are encodings of states.

Our implementation is based on the CUDD package [21, a widely-used and efficient package for the manipulation of MTBDDs. In an MTBDD-based implementation, such as CUDD, the same set of MTBDD variables are used to represent all entities, that is, matrices and sets of states. In our symbolic algorithm, we will need to represent states using either the variable set $v r_{1}, \ldots, v r_{L}$ (row encoding) or $v c_{1}, \ldots, v c_{L}$ (column encoding). We define $\mathcal{B}$ representing a set $B$ such that $\forall t \in S_{c}: \mathcal{B}(s, t)=[s \in B]$ (row encoding) or $\forall t \in S_{r}: \mathcal{B}(t, s)=[s \in B]$ (column encoding), in which $[s \in B]=1$ if $s \in B$ and $[s \in B]=0$ otherwise. Since $\mathcal{B}(s, t)$ (resp. $\mathcal{B}(t, s)$ ) does not depend on $t$ in row (resp. column) encoding we use $\mathcal{B}(s)$ as a shorthand. Permute $(\mathcal{B})$, used in Fig. 1(b), switches the encoding of the set $\mathcal{B}$ from row encoding to column encoding or vice versa. By default, sets are represented using row encoding.

\section{Transforming the Algorithm from Explicit to Symbolic}

To transform the explicit algorithm of Figure 1(a) to a symbolic one, we need to replace both its explicit data structures and also its explicit operations with symbolic counterparts. We already know how to symbolically represent matrices and sets of states. In this section, we first present a new approach for the symbolic representation of partitions. Then, we show how to replace the set of splitters $L$ by partition $\Pi$, thereby representing the set of splitters symbolically. Finally, we explain how the various explicit operations of Figure 1(a) are done symbolically.

\subsection{Symbolic Representation of Partitions}

The challenges in the symbolic representation of partition $\Pi$ are that 1) $|\Pi|$ can be very large, and 2) $\Pi$ is updated frequently during the execution of the algorithm and modifying a symbolic data structure in an "explicit" manner is often very inefficient. Our new symbolic approach for partition representation tries to address these challenges. Of equal importance are its properties that we exploit in Section 4 to improve the running time of our symbolic algorithm. Conceptually, our partition representation technique does not need to be based on a symbolic data structure. However, it will be very inefficient otherwise.

Before we explain our new approach, we give a quick overview of other studied approaches. The first obvious method is to store each class of a partition as a BDD. Another technique, given in [1, is to assign an extra set of BDD variables to denote class indices. In particular, $s \in C_{i}$ iff $\mathcal{P}(s, i)=1$ where $\mathcal{P}$ is the BDD representation of $\Pi$. Yet another approach is to use a BDD $\mathcal{P}$ such that $\mathcal{P}(s, t)=1$ iff $\exists C \in \Pi: s \in C \wedge t \in C$. 
Representation. Let $\Pi=\left\{C_{0}, \ldots, C_{d-1}\right\}$ be a partition of $S \neq \emptyset$. We define a family of sets $\beta(\Pi)=\left\{P_{0}, \ldots, P_{k-1}, S\right\}$ to represent $\Pi$ as follows: $s \in P_{i}$ $(0 \leq i<k)$ iff the $i^{\text {th }}$ bit of the binary representation of the index of $[s]_{\Pi}$ is one. In other words,

$$
P_{i}=\bigcup_{i^{\text {th }} \text { bit of } j \text { is one }} C_{j} \quad \text { and } \quad S-P_{i}=\bigcup_{i^{\text {th }} \text { bit of } j \text { is zero }} C_{j} .
$$

We will use (MT)BDDs to represent members of $\beta(\Pi)$. The important point here is that we can represent $\Pi$ with $k+1=\lceil\lg d\rceil+1$ instead of $d$ (MT)BDDs.

Example. Let $S=\{1, \ldots, 8\}, \Pi=\left\{C_{0}, C_{1}, C_{2}, C_{3}\right\}, C_{0}=\{2,3,8\}, C_{1}=\{1\}$, $C_{2}=\{4,7\}$, and $C_{3}=\{5,6\}$. Then, $\beta(\Pi)=\left\{P_{0}, P_{1}, S\right\}$ in which $P_{0}=\{1,5,6\}$ and $P_{1}=\{4,5,6,7\}$.

Partition Manipulation. In the explicit algorithm, we access $\Pi$ through getting its classes and update $\Pi$ through adding/removing classes to/from it. In the following, we describe how to symbolically perform those manipulations by one access procedure GetClass, and two update procedures RePlaCEClass, and AdDClass. Let $\Pi^{\prime}$ be the modified partition after an update procedure is performed on $\Pi$. Using Eq. (2) to compute the symbolic representation of $\Pi^{\prime}$, i.e., $\beta\left(\Pi^{\prime}\right)$, after each update procedure would take $O\left(2^{k}\right)$ set operations. In the following, we show how to compute it directly from the symbolic representation of $\Pi$, i.e., $\beta(\Pi)$ using only $O(k)$ set operations.

1. GetClass $(\beta(\Pi), j)$ returns $C_{j}$. Let $\left(b_{k-1} \cdots b_{1} b_{0}\right)_{2}$ be the binary representation of $j(0 \leq j<d)$. Then, using Eq. (2), we have

$$
\operatorname{GetClass}(\beta(\Pi), j)=C_{j}=\bigcap_{i=0}^{k-1} D_{i} \quad \text { where } D_{i}= \begin{cases}S-P_{i} & \text { if } b_{i}=0 \\ P_{i} & \text { if } b_{i}=1\end{cases}
$$

Using GetClass, line 1 of ExpSPLit is symbolically performed in lines 1-2 of SyMSplit.

2. ReplaceClass $\left(\beta(\Pi), l, C_{l}^{\prime}\right)$ replaces $C_{l} \in \Pi$ with $C_{l}^{\prime}$ such that $\Pi^{\prime}=(\Pi-$ $\left.\left\{C_{l}\right\}\right) \uplus\left\{C_{l}^{\prime}\right\}=\left\{C_{0}, \ldots, C_{l-1}, C_{l}^{\prime}, C_{l+1}, \ldots C_{d-1}\right\}$. We have $S^{\prime}=\left(S-C_{l}\right) \uplus$ $C_{l}^{\prime}$, and by Eq. (2),

$$
P_{i}^{\prime}= \begin{cases}P_{i} & \text { if } i \text { th bit of } l \text { is zero } \\ \left(P_{i}-C_{l}\right) \uplus C_{l}^{\prime} & \text { if } i \text { th bit of } l \text { is one }\end{cases}
$$

3. $\operatorname{AddClass}\left(\beta(\Pi), C_{d}\right)$ adds $C_{d}$ to $\Pi$ where $C_{d}$ is non-empty set disjoint with all members of $\Pi$. Obviously, we have $S^{\prime}=S \uplus C_{d}$, and by Eq. (2),

$$
P_{i}^{\prime}= \begin{cases}P_{i} & \text { if } i \text { th bit of } d \text { is zero } \\ P_{i} \uplus C_{d} & \text { if } i \text { th bit of } d \text { is one }\end{cases}
$$

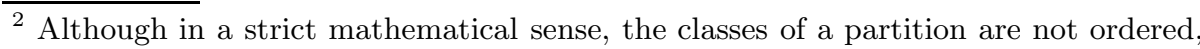
we assign them a total order here.

${ }^{3} \uplus$ is the disjoint union operation.
} 
For $d=2^{k}$, assume $P_{k}=\emptyset$. Using REPLACECLASs and a sequence of ADDCLASS operations, line 4 of ExPSPLIT is symbolically performed in lines 9-11 of SyMSPLIT.

\subsection{Replacing Explicit $L$ by Symbolic $\Pi$}

In our new symbolic algorithm, we need to have a symbolic representation of $L$ that is efficient to update. Knowing that (1) similar to $\Pi, L$ is a set of sets of states, and (2) updates of $L$ is very similar to updates of $\Pi$ (compare lines 4 and 5 of ExPSPLIT), we will show how we have modified our algorithm such that we do not need to explicitly store $L$. Instead, we use the symbolic representation of $\Pi$ and an index to emulate a list of potential splitters.

Consider Fig. 1. We have removed $L$ from ExpCoARsESTPART (lines 2-5) and ExpSplit (line 5) and replaced it by an index $s c$ in SyMCoARSESTPART (lines 2-3). In line 2 of SyMCOARSESTPART, $s c$ iterates through all classes of $\Pi$. During the running time of the algorithm, classes are possibly added to (the end of) $\Pi$. Therefore, $\mathcal{B}$, in line 3 of SyMCOARSESTPART, will take on the value of all those new classes, one at a time. Note that the set of potential splitters processed by SymSplit may be different from the one processed by ExpSpLit. However, we will prove that SYMCOARSESTPART still works correctly by showing that the different sets of splitters that ExPCOARSESTPART and SymCoARsESTPART see have the same refinement effect on $\Pi$.

Lemma 1. Assume $C \subseteq S$ and $\left\{C_{1}^{\prime}, \ldots, C_{\alpha}^{\prime}\right\}$ be a partition of $C$. Then, splitting a partition $\Pi$ of $S$ with respect to any $\alpha$ members of $T=\left\{C, C_{1}^{\prime}, \ldots, C_{\alpha}^{\prime}\right\}$ leads to the same refinement of $\Pi$.

Proof. We give the proof for ordinary lumping. The arguments for exact lumping are similar. According to Eq. (1), for any splitter $B \in T, \mathbf{R}(s, B)$ determines how the blocks of $\Pi$ are partitioned. Moreover, for any state $s \in S$, we have $\mathbf{R}\left(s, C_{1}^{\prime}\right)+\cdots+\mathbf{R}\left(s, C_{\alpha}^{\prime}\right)=\mathbf{R}(s, C)$. Therefore, given any $\alpha$ terms of the equality, the $(\alpha+1)$-st term is implicit. Hence, splitting with respect to any $B \in T$ does not further refine a partition that has already been refined with respect to the other $\alpha$ members of $T$.

Theorem 3. The sequence of splitters seen by SyMCOARSESTPART leads to the correct refinement of $\Pi$.

Proof. We need to show that each time a block $\mathcal{C}$ is partitioned into $\mathcal{C}_{1}^{\prime}, \ldots, \mathcal{C}_{\alpha}^{\prime}$, at least $\alpha$ members of $\left\{\mathcal{C}, \mathcal{C}_{1}^{\prime}, \ldots, \mathcal{C}_{\alpha}^{\prime}\right\}$ have already been or will be seen by SYMCoArsestPart. Assume the algorithm is at the beginning of line 9 of SYMSPLiT. There are two cases. If $s c \leq c$, then SymCoARSESTPART has not yet seen $\mathcal{C}$ as a splitter, and lines 9-11 replace $\mathcal{C}$ with $\left\{\mathcal{C}_{1}^{\prime}, \ldots, \mathcal{C}_{\alpha}^{\prime}\right\}$. All those $\alpha$ sets will be seen as splitters in future iterations of SYMCOARSESTPART. If $s c>c$, then SyMCOARSESTPART has already used $\mathcal{C}$ as a splitter, and lines 9-11 add $\alpha-1$ sets, i.e., $\left\{\mathcal{C}_{2}^{\prime}, \ldots, \mathcal{C}_{\alpha}^{\prime}\right\}$, to the end of $\Pi$. All of those sets will be seen by SYMCOARSESTPART in its future iterations. 


\subsection{Symbolic Procedures: SymComputeKeys and SymSplit}

Let $B, C \subseteq S$. ExpComputeKeys computes $\mathbf{R}(s, B)$ for ordinary lumping and $\mathbf{R}(B, s)$ for exact lumping for each $s \in C$. In order to compute $\mathbf{R}(s, B)$ and $\mathbf{R}(B, s)$ symbolically, we define $\mathcal{R}_{o}^{C, B}$ and $\mathcal{R}_{e}^{C, B}$ as follows:

$$
\begin{array}{llll}
\mathcal{R}_{o}^{C, B}(s, t)=r \text { iff } & \mathcal{R}(s, t)=r \wedge \mathcal{C}(s)=1 \wedge \mathcal{B}(t)=1 & & \text { (ordinary lumping) } \\
\mathcal{R}_{e}^{C, B}(s, t)=r \text { iff } & \mathcal{R}(s, t)=r \wedge \mathcal{B}(s)=1 \wedge \mathcal{C}(t)=1 & & \text { (exact lumping) }
\end{array}
$$

In other words, $\mathcal{R}_{o}^{C, B}$ (resp. $\mathcal{R}_{e}^{C, B}$ ) is the same as $\mathcal{R}$ except that its set of rows and columns are restricted to $\mathcal{C}$ and $\mathcal{B}$ (resp. $\mathcal{B}$ and $\mathcal{C}$ ) respectively. $\mathcal{R}_{o}^{C, B}$ and $\mathcal{R}_{e}^{C, B}$ are computed in lines $1_{o}$ and $1_{e}$ of SymComputeKeys, the symbolic version of ExpComputeKeys. SymComputeKeys uses Apply $(\bowtie, \mathcal{X}, \mathcal{Y})$ which is provided by the CUDD package and returns an MTBDD $\mathcal{Z}$ such that $\mathcal{Z}(s, t)=\mathcal{X}(s, t) \bowtie \mathcal{Y}(s, t)$ where $\bowtie$ is an arithmetic or logical operator. For logical operators, APPLY returns an MTBDD with only 0 and 1 terminals.

Now, we define MTBDDs $\mathcal{K}_{o}^{C, B}(s, t)$ and $\mathcal{K}_{e}^{C, B}(s, t)$ as follows:

$$
\begin{aligned}
& \forall t \in S_{c}: \mathcal{K}_{o}^{C, B}(s, t)=\sum_{t^{\prime} \in S_{c}} \mathcal{R}_{o}^{C, B}\left(s, t^{\prime}\right)=\sum_{t^{\prime} \in B} \mathcal{R}_{o}^{C, B}\left(s, t^{\prime}\right)=\mathbf{R}(s, B) \\
& \forall s \in S_{r}: \mathcal{K}_{e}^{C, B}(s, t)=\sum_{s^{\prime} \in S_{r}} \mathcal{R}_{e}^{C, B}\left(s^{\prime}, t\right)=\sum_{s^{\prime} \in B} \mathcal{R}_{e}^{C, B}\left(s^{\prime}, t\right)=\mathbf{R}(B, t) .
\end{aligned}
$$

Since $\mathcal{K}_{o}^{C, B}(s, t)=\mathbf{R}(s, B)$ and $\mathcal{K}_{e}^{C, B}(s, t)=\mathbf{R}(B, t), \mathcal{K}_{o}^{C, B}$ and $\mathcal{K}_{e}^{C, B}$ are in fact the MTBDD representations of $\mathbf{k}$ in Section 2.2. Thus, they are the key to partition $\mathcal{C}$ into $\left\{\mathcal{C}_{1}^{\prime}, \ldots, \mathcal{C}_{\alpha}^{\prime}\right\}$ according to Eq. (1). They are computed symbolically using SumC and SumR. For an $\operatorname{MTBDD} \mathcal{A}, \operatorname{SumC}(\mathcal{A})$ returns $\mathcal{A}^{\prime}$ such that $\forall t \in S_{c}: \mathcal{A}^{\prime}(s, t)=\sum_{t^{\prime} \in S_{c}} \mathcal{A}\left(s, t^{\prime}\right)$. Similarly, $\operatorname{SumR}(\mathcal{A})$ returns $\mathcal{A}^{\prime}$ such that $\forall s \in S_{r}: \mathcal{A}^{\prime}(s, t)=\sum_{s^{\prime} \in S_{r}} \mathcal{A}\left(s^{\prime}, t\right)$. SumC and SumR are implemented using Cudd_addExistAbstract function of the CUDD package. Lines 4-8 of SYMSPLIT show how to symbolically derive $\mathcal{C}_{1}^{\prime}, \ldots, \mathcal{C}_{\alpha}^{\prime}$ from $\mathcal{C}$. Line 4 is done using a depth first traversal of $\mathcal{K}$.

Note that if there is no transition from any state in $\mathcal{C}$ (resp. $\mathcal{B}$ ) to any state in $\mathcal{B}$ (resp. $\mathcal{C}$ ), then $\mathcal{R}_{o}^{C, B}\left(\right.$ resp. $\left.\mathcal{R}_{e}^{C, B}\right)$, and therefore, $\mathcal{K}_{o}^{C, B}$ (resp. $\mathcal{K}_{e}^{C, B}$ ) are zerovalued MTBDDs. Hence, $\mathcal{C}$ will not be split. The second technique in Section 4 exploits that observation to improve the running time of the symbolic algorithm.

So far, we have transformed all data structures and operations of the explicit procedures of Figure 1(a) to symbolic ones in their corresponding symbolic procedures. That gives us the completely symbolic algorithm of Figure 1(b).

\section{Improving the Symbolic Algorithm Running Time}

The properties of our partition representation method enable us to improve the running time of the symbolic algorithm developed in Section 3. In this section, we present two techniques T1 and T2 that utilize those properties. Both techniques use relatively small additional memory to gain significant speed improvements. 
Based on the combination of the techniques, we distinguish three versions of our algorithm: V1 uses neither techniques, V2 uses T1 only, and V3 uses both $\mathrm{T} 1$ and $\mathrm{T} 2$. In the following, we present the arguments only for ordinary lumping and they are straightforwardly adaptable to the case of exact lumping. We will compare the performance of the three versions in Section 5 .

\subsection{T1: Computing $\operatorname{Get} \operatorname{Class}(\beta(\Pi), j+1)$ from $\operatorname{GetClass}(\beta(\Pi), j)$}

The main loops of SymCOARSESTPART and SYMSPLIT compute all classes of $\Pi$ using GeTCLASS. T1 is an algorithm that computes those classes more efficiently than naively applying Eq. (3) for each class. To do so, T1 exploits the similarity between the computation of all pairs of consecutive classes of $\Pi$.

Figure 2 shows the Class Computation Tree (CCT) for $\Pi$. The tree shows the sequence of set operations that GETCLAss executes for all classes of $\Pi$ according to Eq. (3) 3 . It has $k+1$ levels numbered top-down from 0 to $k$, and hence, has at most $2^{k+1}-1$ nodes. We denote the root node by $r$. A non-root node $v \neq r$ is connected to its parent $p(v)$ by an edge with label $e(v) \subseteq S$. For a non-leaf node $u$ at level $i$, we denote its left and right children by $u_{l}$ and $u_{r}$. We define $e\left(u_{l}\right)=S-P_{i}$ and $e\left(u_{r}\right)=P_{i}$. A path from $r$ to $v$ corresponds to a set expression $E(v)$ defined recursively as follows:

$$
E(r)=S, \text { and } E(v)=E(p(v)) \cap e(v) .
$$

For example, in Fig. 2, we have $E\left(u^{\prime}\right)=S \cap\left(S-P_{0}\right)=\left(S-P_{0}\right)$ and $E\left(v_{0}\right)=$ $S \cap\left(S-P_{0}\right) \cap \ldots \cap\left(S-P_{k-1}\right)$. Indexing the leaf nodes from left to right starting from 0 , we observe that for a leaf node $v_{j}$ with index $j, E\left(v_{j}\right)$ yields the $j$ th class of $\Pi$, i.e., $E\left(v_{j}\right)=C_{j}=\operatorname{GetClass}(\beta(\Pi), j)$.

For any leaf node $v$, the number of set intersections in $E(v)$, and hence, the number of times GeTCLASS performs set intersection, is $k$. Hence, calling GetClass for all classes of $\Pi$ requires $k \cdot d=\Theta(d \lg d)$ set intersections in which $d=|\Pi|$.

Now consider two classes $C_{j}, C_{j^{\prime}} \in \Pi\left(j^{\prime}=j+1\right.$ is a special case $)$ and their corresponding leaf nodes $v_{j}$ and $v_{j^{\prime}}$ (See Figure 2). We observe that set expressions $E\left(v_{j}\right)$ and $E\left(v_{j^{\prime}}\right)$ have a common prefix subexpression which is determined by the lowest common ancestor node $x$ of $v_{j}$ and $v_{j^{\prime}}$. Hence, by storing $E(x)$ at $x$ during the computation of $C_{j}=E\left(v_{j}\right)$, we can compute $C_{j^{\prime}}=E\left(v_{j^{\prime}}\right)$ with smaller number of set intersections than what would be necessary for its computation from scratch using Eq. (3).

Making the above observation, we propose the following method to compute all classes of $\Pi$ in order of their indices: perform a depth first traversal of the $\mathrm{CCT}$ such that the left subtree is visited before the right subtree. At each nonroot node $v$ compute $E(v)$ using Eq. (4) and store it as an MTBDD. The number of set intersections performed is the number of edges of the CCT which is at most $2^{k+1}-2 \in \Theta(d)$. Note that we do not need to store $E(v)$ for all nodes; storing one per level suffices.

In summary, $\mathrm{T} 1$ reduces the number of set operations necessary to compute all classes of $\Pi$ from $\Theta(d \lg d)$ to $\Theta(d)$ using $k+1$ extra MTBDDs.

\footnotetext{
${ }^{4}$ The tree is not generated or stored by the algorithm.
} 


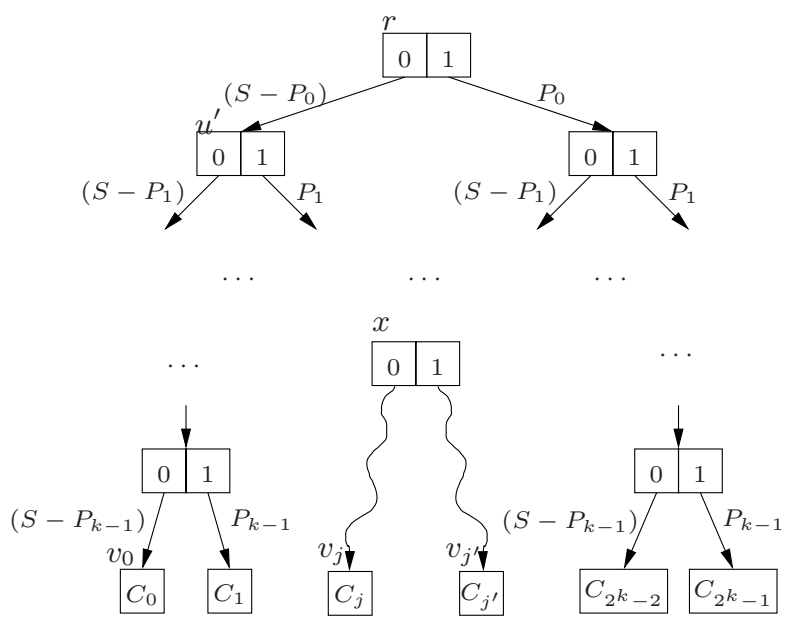

Fig. 2. Class Computation Tree (CCT)

\subsection{T2: Fast Detection and Skipping of Stable Classes}

SymSplit splits each class $\mathcal{C} \in \Pi$ with respect to the splitter $\mathcal{B}$. However, if there is no transition from any state in $\mathcal{C}$ to any state in $\mathcal{B}$, then $\mathcal{C}$ will not be split into smaller subclasses. Therefore, executing lines 2-11 of SyMSPLIT can be skipped for classes such as $\mathcal{C}$. We call $\mathcal{C}$ stable with respect to $\mathcal{B}$.

$\mathrm{T} 2$ is a technique for efficient detection of stable classes. It enables the main loop of SyMSPLIT to skip over those classes, thereby reducing SYMSPLIT's running time. CTMCs generated from high-level models often have sparse transition matrices. For such CTMCs, the ratio of stable classes (with respect to a given $\mathcal{B})$ to the total number of classes is often close to 1 . Therefore, T2 yields a considerable speedup.

Let $B^{\prime}$ be the set of states that have at least one transition to any state in $B$, i.e., $B^{\prime}=\left\{s^{\prime} \mid \exists s \in B, \mathbf{R}\left(s^{\prime}, s\right) \neq 0\right\}$. Observe that $C$ is stable with respect to $B$ iff $B^{\prime} \cap C=\emptyset$. Therefore, the problem is reduced to evaluating whether $B^{\prime} \cap C=\emptyset$. If $B^{\prime}=\emptyset$ every class $C \in \Pi$ is stable with respect to $B$. In the following, we assume that $B^{\prime} \neq \emptyset$.

Using the modified $C C T$, a slight modification of the CCT, we can efficiently compute $B^{\prime} \cap C$ for all $C \in \Pi$. The modified CCT uses the following equation to compute the set expression $E^{\prime}(v)$ corresponding to a node $v: E^{\prime}(r)=B^{\prime}$ and $E^{\prime}(v)=E^{\prime}(p(v)) \cap e(v)$ for $v \neq r$. Thus, $E^{\prime}\left(v_{j}\right)=B^{\prime} \cap C_{j}$ for a leaf node $v_{j}$ with index $j$. Finally, checking for emptiness of an MTBDD takes constant time.

A significant improvement is achieved by observing that if $E^{\prime}(v)$ is empty, so is $E^{\prime}\left(v^{\prime}\right)$ for all descendants $v^{\prime}$ of $v$. Thus, we can prune the tree at node $v$, thereby saving time on its traversal. 


\section{Performance Study}

While the previous sections show that our symbolic algorithm is efficient from a theoretical point of view, the evidence of its utility comes from its implementation and use on example models. In this section, we briefly describe the implementation we have made, and compare its performance with implementations of other related algorithms. The performance measures that we compare are mainly the time and space requirements of the algorithms and the size of lumped MCs that they generate.

In particular, we compare the performance of the different versions of the algorithm described in Section 4 (that is, V1, V2, and V3), the state-level explicit algorithm (EA) of 9, and the model-level symbolic algorithm of Kwiatkowska et. al. 18, (KA). Our experiments on two example models show that (1) our symbolic algorithm is able to lump MCs that are orders of magnitude larger than what is lumpable using an explicit lumping algorithm, (2) the techniques explained in Section 4 reduce the running time of the symbolic algorithm by up to 3 orders of magnitude, (3) in the best case we tried, V3, the fastest version of our symbolic algorithm outperforms EA, and in the worst case, it is not prohibitively slower than EA, and (4) KA is a few orders of magnitude faster than V3 while V3 generates lumped MCs that are (sometimes several) orders of magnitude smaller.

\subsection{Implementation and Example Models}

To generate both the MTBDD and sparse matrix representations of the input Markov chains, we use the probabilistic model checking tool PRISM [15]. All the code involved in the experiments was compiled using gcc 3.4.4. All experiments were conducted on a Pentium $42.66 \mathrm{GHz}$ CPU with $1 \mathrm{~GB}$ of RAM.

We consider two example models from the literature to study the performance of the algorithms: A fault-tolerant parallel computer system (FPCS) [19] and a peer-to-peer (P2P) protocol based on BitTorrent 18. For the first model, we converted the SAN (Stochastic Activity Network) specification to the PRISM specification. For the second model, we used the PRISM specification given in http://www.cs.bham.ac.uk/ dxp/prism/casestudies/peer2peer.php.

Both models have two parameters $N_{1}$ and $N_{2}$. For FPCS, they denote the number of computers in the system and the number of memory modules in each computer, respectively. For P2P, they represent the number of clients and the number of blocks of the file to be transmitted, respectively.

\subsection{Results}

Comparison of V1, V2, V3, and EA. EA is theoretically the fastest explicit state-level lumping algorithm given so far 5 . We applied the ordinary lumping algorithm of V1, V2, V3, and EA on a number of configurations of FPCS and

\footnotetext{
${ }^{5}$ We are not aware of a study that compares the practical performance of various explicit state-level algorithms.
} 
Table 2. Performance Results

(a) Performance comparison of symbolic and explicit algorithms

\begin{tabular}{|c|c|c|c|c|c|c|c|c|c|c|c|c|}
\hline & \multirow{2}{*}{$\begin{array}{c}\text { Config } \\
\left(N_{1}, N_{2}\right)\end{array}$} & \multicolumn{2}{|c|}{ \# of states } & \multicolumn{2}{|c|}{ \# of nodes } & \multicolumn{4}{|c|}{ total running time $(\mathrm{sec})$} & \multicolumn{3}{|c|}{ peak \# of nodes } \\
\hline & & $n$ & $\bar{n}$ & $\eta$ & $\bar{\eta}$ & V1 & $\mathrm{V2}$ & $V 3$ & EA & V1 & 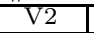 & V3 \\
\hline \multirow{7}{*}{ 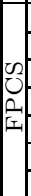 } & $(2,2)$ & $1.58 e 4$ & 703 & 5960 & 4979 & $1.83 e 2$ & $4.00 e 1$ & $7.50 e 0$ & $5.20 e-1$ & $2.58 e 4$ & $2.58 e 4$ & $2.75 e 4$ \\
\hline & $(3,1)$ & $2.30 e 4$ & 969 & 14370 & 9079 & $1.00 e 3$ & $1.90 \mathrm{e} 2$ & $2.90 e 1$ & $8.80 e-1$ & $6.42 e 4$ & $6.42 e 4$ & $6.86 e 4$ \\
\hline & $(2,3)$ & $2.57 e 5$ & 2145 & 9114 & 13731 & $1.20 e 4$ & $1.50 e 3$ & $7.80 e 1$ & $1.00 e 1$ & $6.82 e 4$ & $6.82 e 4$ & $7.09 e 4$ \\
\hline & $(3,2)$ & $1.89 e 6$ & 9139 & 34122 & 43134 & TL & TL & $4.20 e 3$ & $9.40 e 1$ & $\overline{T L}$ & $4.68 e 5$ & $4.78 e 5$ \\
\hline & $(2,4)$ & $3.80 e 6$ & 5151 & 12314 & 34318 & TL & $2.60 e 4$ & $5.80 e 2$ & $1.80 e 2$ & TL & $1.62 e 5$ & $1.65 e 5$ \\
\hline & $(2,5)$ & $5.26 e 7$ & 10585 & 15468 & 70809 & TL & $2.30 e 5$ & $3.00 e 3$ & ML & TL & $3.49 e 5$ & $3.54 e 5$ \\
\hline & $(3,3)$ & $1.24 e 8$ & 47905 & 53177 & 151368 & TL & TL & $1.15 e 5$ & $\mathrm{ML}$ & $\overline{T L}$ & $\mathrm{TL}$ & $2.56 e 6$ \\
\hline \multirow{6}{*}{ ît } & $(3,5)$ & $3.28 e 4$ & 56 & 2451 & 1751 & $3.40 e 0$ & $2.72 e 0$ & $1.70 e 0$ & $8.38 e-1$ & $2.36 e 4$ & $2.73 e 4$ & $2.83 e 4$ \\
\hline & $(4,5)$ & $1.05 e 6$ & 126 & 11941 & 5914 & $8.04 e 1$ & $5.17 e 1$ & $2.03 e 1$ & $3.84 e 1$ & $1.18 e 5$ & $1.43 e 5$ & $1.50 e 5$ \\
\hline & $(5,5)$ & $3.36 e^{7}$ & 196 & 26266 & 10975 & $7.43 e 2$ & $4.13 e 2$ & $1.37 e 2$ & ML & $3.63 e 5$ & $4.44 e 5$ & $4.68 e 5$ \\
\hline & $(6,5)$ & $1.07 e 9$ & 266 & 40591 & 20212 & $3.64 e 3$ & $1.91 e 3$ & $5.56 e^{2}$ & ML & $8.56 e 5$ & $1.06 e 6$ & $1.12 e 6$ \\
\hline & $(7,5)$ & $.44 e 10$ & 336 & 54916 & 26182 & $1.18 e 4$ & $6.22 e 3$ & $1.64 e 3$ & ML & $1.54 \mathrm{e} 6$ & $1.83 e 6$ & $1.93 e 6$ \\
\hline & $(8,5)$ & $1.10 e 11$ & 406 & 69241 & 36153 & $4.43 e 4$ & $2.53 e 4$ & $1.14 e 4$ & ML & $2.65 e 6$ & $3.37 e 6$ & $3.51 e 6$ \\
\hline
\end{tabular}

(b) Comparison of V3, Kwiatkowska's algorithm and their combination

\begin{tabular}{|c|c|c|c|c|c|c|c|c|c|c|}
\hline \multirow{2}{*}{ Model } & \multirow{2}{*}{$\begin{array}{c}\text { Config } \\
\left(N_{1}, N_{2}\right)\end{array}$} & \multicolumn{3}{|c|}{ \# of states } & \multicolumn{3}{|c|}{ \# of nodes } & \multicolumn{3}{|c|}{ running times (sec) } \\
\hline & & $n$ & $\bar{n}_{\mathrm{KA}}$ & $n_{\mathrm{V} 3}$ & $\eta$ & $\eta_{\mathrm{KA}}$ & $\eta_{\mathrm{V} 3}$ & V3 & KA & Comb. \\
\hline \multirow{6}{*}{$\mathrm{P} 2 \mathrm{P}$} & $(3,5)$ & $3.28 e 4$ & $5.98 e 3$ & 56 & 2451 & 12518 & 1751 & $1.70 e 0$ & $1.15 e-1$ & $2.15 e 0$ \\
\hline & $(4,5)$ & $1.05 e 6$ & $5.24 e 4$ & 126 & 11941 & 42166 & 5914 & $2.03 e 1$ & $4.90 e-1$ & $2.56 e 1$ \\
\hline & $(5,5)$ & $3.36 e^{7}$ & $3.77 e 5$ & 196 & 26266 & 101630 & 10975 & $1.37 e 2$ & $1.30 e 0$ & $1.68 e 2$ \\
\hline & $(6,5)$ & $1.07 e 9$ & $2.32 e 6$ & 266 & 40591 & 189704 & 20212 & $5.56 e 2$ & $3.05 e 0$ & $7.09 e 2$ \\
\hline & $(7,5)$ & $3.44 e 10$ & $1.26 e^{7}$ & 336 & 54916 & 306123 & 26182 & $1.64 e 3$ & $\overline{5.11 e 0}$ & $2.26 e 3$ \\
\hline & $(8,5)$ & $1.10 \mathrm{e} 11$ & $6.15 e 7$ & 406 & 69241 & 449599 & 36153 & $1.14 e 4$ & $9.17 e 0$ & $1.48 e 4$ \\
\hline
\end{tabular}

P2P. The results are given in Table 2(a) Columns 3 to 6 give the number of states and MTBDD nodes of the original (input) and the lumped (output) MCs. Times shown in columns 7 to 10 include both the partition computation and the quotient construction times. The last three columns give the maximum number of live MTBDD nodes during the runtime of V1, V2, and V3. ML (Memory Limit) and TL (Time Limit) mean that the corresponding data is not available because the algorithm ran out of memory and its running time exceeded 3 days $\left(\approx 2.5 \times 10^{5}\right.$ seconds $)$, respectively.

Since all algorithms are optimal, they generate the same lumped MCs, and for V1-V3, with the same MTBDD representations. It has been observed (e.g., see [13] ) that lumping often increases the size of the MTBDD representation, i.e., $\eta<$ $\widetilde{\eta}$. The reason is that the structure regularity of the MTBDD of the lumped MC is lost. In our experiments, that holds true when $\widetilde{n}$ is sufficiently large.

From Table 2(a) we can see how effective T1 and T2, the improvement techniques described in Section 4, are. Based on all experiments, V3 is faster than $\mathrm{V} 2$ by a factor of 1.6 to 76 and V2 is faster than V1 by a factor of 1.3 to 10 . Since $\mathrm{T} 1$ saves time on computing all classes of $\Pi$ and $\mathrm{T} 2$ does so by skipping stable classes of $\Pi$, their effects grow as $|\Pi|$ and $\tilde{n}$ increase (note that $|\Pi| \leq \widetilde{n}$ during the runtime of the algorithm). That is the reason why the speedup factors are less for P2P than FPCS and for each model the speedup factors increase as $\widetilde{n}$ grows. Overall, the combination of $\mathrm{T} 1$ and $\mathrm{T} 2$ achieve a speedup of 2 to 700, depending on the input MC. Note that their combined memory overhead, in 
terms of the number of alive nodes, is very low (at most $32 \%$ ) relative to the speedup they cause.

Those improvements significantly pale the speed disadvantage that V3 has compared to EA. We observed that if the structure of input MTBDD is sufficiently regular and the CTMC is significantly lumpable, V3 outperforms EA. In configuration $(4,5)$ of $\mathrm{P} 2 \mathrm{P}, \mathrm{V} 3$ is 1.9 times faster than EA. We anticipate that the ratio of V3's speed to EA's would increase for larger P2P models if EA did not run out of memory. The reason is that V3's running time is growing slower than $n$ while EA's would increase at least as fast as $n$. Although V3 is 45 times slower than EA on one of the experiments, its main advantage comes from its ability to handle MCs that are several orders of magnitude larger.

SymCompQuOT of Fig. 1(b) has two explicit loops over states of the lumpable partition. Therefore, one may not consider it as a "very symbolic" algorithm or may have suspicion about its efficiency. Based on our measurements (not shown in Table 2(a)p, SymCompQuot never takes more than $23 \%$ of the total running time of the symbolic algorithm for the FPCS model. The corresponding number for the $\mathrm{P} 2 \mathrm{P}$ model is $6 \%$.

Comparison and Combination of Two Symbolic Algorithms. Finally, we compare the performance of V3 against another symbolic algorithm. We are not aware of any other state-level symbolic lumping algorithm. However, we think that it is informative to compare our algorithm to the MTBDD-based model-level lumping algorithm of Kwiatkowska et. al. [18]. Kwiatkowska's algorithm (KA) exploits a special type of symmetry, i.e., symmetry among identical components.

Table 2(b) shows the results of our experiments with V3 and KA 6 . In general, $\widetilde{n}_{\mathrm{KA}} \neq \widetilde{n}_{\mathrm{V} 3}$ since $\mathrm{KA}$ is not optimal, and therefore, may not generate the smallest quotient CTMC for all inputs. Based on the results given in Table 2(b) we observe that (1) KA is a few orders of magnitude faster than our algorithm because it gets symmetry information from the high-level specification of the model and not from the CTMC, (2) $\widetilde{n}_{\mathrm{V} 3}$ is (sometimes, several) orders of magnitude smaller than $\widetilde{n}_{\mathrm{KA}}$ because V3 is optimal, (3) V3 may additionally lead to a much smaller MTBDD representation $\left(\widetilde{\eta}_{\mathrm{KA}} \gg \widetilde{\eta}_{\mathrm{V} 3}\right)$ as is the case for all instances of P2P model we tried. Obviously, (2) generally holds for models that are lumpable due to symmetries other than those exploited by KA. For models that have no symmetries but those exploitable by KA, KA is much more efficient than V3 in that it would generate the (same) smallest quotient MC much faster.

In explicit lumping algorithms, we observe the same trend when comparing state-level and model-level algorithms: the former are slower but may generate much smaller quotient CTMCs. Since the running time of explicit state-level algorithms are at least linear in $n$, it will be beneficial to combine the the statelevel and the model-level algorithms, i.e., to apply them in sequence. First, the

\footnotetext{
${ }^{6}$ We did not include the FPCS model in Table 2(b) because KA does not currently support exploiting the hierarchical symmetries of the FPCS model. However, we believe that the theory and implementation of KA are extendible to hierarchical symmetries in a straightforward manner as in 10 .
} 
model-level algorithm quickly produces a partially lumped CTMC. Then, the state-level algorithm takes the result and produces the optimally lumped CTMC much faster than what it would take the state-level algorithm to optimally lump the original CTMC.

The last column of Table 2(b) shows the total running time of applying KA and V3 in sequence. As we can see, in the case of symbolic algorithms, the combination is always slower than V3. That is not a surprising result because the running time of a symbolic state-level algorithm (e.g., V3) does not depend on the size of the state space of the input CTMC. Rather, it depends on the structure regularity and the number of nodes of the input MTBDD; the former is diminished and the latter is increased by the model-level algorithm $\left(\widetilde{\eta}_{\mathrm{KA}} \gg \eta\right)$.

\section{Conclusion and Future Work}

In this paper, we developed the first symbolic state-level lumping algorithm for Markov chains using a new partition representation technique whose properties enabled us to improve the running time of the algorithm by up to three orders of magnitude. In the worst case we experimented, our symbolic algorithm was less than two orders of magnitude slower than an efficient explicit algorithm. In the best case, the former was even faster by a factor of 1.9. The natural strength of our algorithm is its ability to lump CTMCs with state spaces that are several orders of magnitude larger than what the explicit algorithm can.

We also compared our state-level symbolic algorithm with Kwiatkowska's symbolic model-level algorithm. We observed in our experiments that although our algorithm is a few orders of magnitude slower, it generates lumped CTMCs that are several orders of magnitude smaller. Finally, we combined the two symbolic algorithms. Unlike the explicit case, the combination is always slower than the state-level algorithm due to loss of structure regularity and increase in size of the MTBDD representation by the model-level algorithm.

There is no study that shows the effect of the various partition representation methods on the performance of (Markov chain) lumping algorithms. This paper is a first step toward that study. We also would like to investigate whether our partition representation method benefits other symbolic algorithms. Finally, we intend to integrate the algorithm into PRISM.

Acknowledgments. We would like to thank Holger Hermanns for pointing out some of the previous work, Dave Parker, Gethin Norman, and Marta Kwiatkowska for their technical support with the PRISM tool and and the P2P model, Shravan Gaonkar for his helpful comments on the manuscript, and last but not least, the reviewers for their very useful feedback.

\section{References}

1. E. Böde, M. Herbstritt, H. Hermanns, S. Johr, T. Peikenkamp, R. Pulungan, R. Wimmer, and B. Becker. Compositional performability evaluation for STATEMATE. In Proc. of QEST, USA, Sep. 2006. 
2. A. Bouali and R. de Simone. Symbolic bisimulation minimisation. In Proc. of $C A V$, volume 663 of $L N C S$, pages 96-108. Springer, 1992.

3. R. E. Bryant. Graph-based algorithms for Boolean function manipulation. IEEE Trans. Comp., 35(8):677-691, Aug. 1986.

4. P. Buchholz. Exact and ordinary lumpability in finite Markov chains. Journal of Applied Probability, 31:59-74, 1994.

5. P. Buchholz. Efficient computation of equivalent and reduced representations for stochastic automata. Int. Journal of Comp. Sys. Sci. ES Eng., 15(2):93-103, 2000.

6. G. Ciardo and A. S. Miner. A data structure for the efficient Kronecker solution of GSPNs. In Proc. of PNPM, pages 22-31, 1999.

7. E. Clarke, M. Fujita, P. McGeer, K. McMillan, J. Yang, and X. Zhao. Multiterminal binary decision diagrams: An efficient data structure for matrix representation. Formal Methods in System Design, 10(2/3):149-169, 1997.

8. S. Derisavi. Solution of Large Markov Models Using Lumping Techniques and Symbolic Data Structures. PhD thesis, U. of Illinois at Urbana-Champaign, 2005.

9. S. Derisavi, H. Hermanns, and W. H. Sanders. Optimal state-space lumping in Markov chains. Information Processing Letters, 87(6):309-315, September 2003.

10. S. Derisavi, P. Kemper, and W. H. Sanders. Symbolic state-space exploration and numerical analysis of state-sharing composed models. Linear Algebra and Its Applications, 386:137-166, July 15, 2004.

11. S. Derisavi, P. Kemper, and W. H. Sanders. Lumping matrix diagram representations of markovian models. In Proc. of DSN, pages 742-751, Japan, 2005.

12. H. Hermanns. Interactive Markov Chains and the Quest for Quantified Quality, volume 2428 of $L N C S$. Springer, 2002.

13. H. Hermanns, J. Meyer-Kayser, and M. Siegle. Multi terminal binary decision diagrams to represent and analyse continuous time Markov chains. In Proc. of 3rd Meeting on Numerical Solution of Markov Chains (NSMC), pages 188-207, 1999.

14. H. Hermanns and M. Siegle. Bisimulation algorithms for stochastic process algebras and their bdd-based implementation. In ARTS, pages 244-264, 1999.

15. A. Hinton, M. Kwiatkowska, G. Norman, and D. Parker. PRISM: A tool for automatic verification of probabilistic systems. In H. Hermanns and J. Palsberg, editors, Proc. of TACAS '06, volume 3920 of LNCS, pages 441-444. Springer, 2006.

16. R. A. Howard. Dynamic Probabilistic Systems, Volume II: Semi-Markov and Decision Processes. Wiley, New York, 1971.

17. J. G. Kemeney and J. L. Snell. Finite Markov Chains. D. Van Nostrand Company, Inc., 1960.

18. M. Kwiatkowska, G. Norman, and D. Parker. Symmetry reduction for probabilistic model checking. In T. Ball and R. Jones, editors, Proc. of $C A V$, volume 4114 of LNCS, pages 234-248. Springer-Verlag, 2006.

19. W. H. Sanders and L. M. Malhis. Dependability evaluation using composed SANbased reward models. J. of Para. and Dist. Comp., 15(3):238-254, July 1992.

20. W. H. Sanders and J. F. Meyer. Reduced base model construction methods for stochastic activity networks. IEEE J. on Selected Areas in Comm., 9(1):25-36, Jan. 1991.

21. F. Somenzi. CUDD: Colorado University decision diagram package. public software, Colorado Univeristy, Boulder, http://vlsi.colorado.edu/ fabio/.

22. R. Wimmer, M. Herbstritt, H. Hermanns, K. Strampp, and B. Becker. Sigref - a symbolic bisimulation tool box. In Proc. of ATVA '06, China, 2006. to appear. 